\title{
Combinatorial Hopf algebras from renormalization
}

\author{
Christian Brouder • Alessandra Frabetti • \\ Frédéric Menous
}

Received: 17 September 2009 / Accepted: 29 March 2010 / Published online: 1 May 2010

(C) Springer Science+Business Media, LLC 2010

\begin{abstract}
In this paper we describe the right-sided combinatorial Hopf structure of three Hopf algebras appearing in the context of renormalization in quantum field theory: the non-commutative version of the Faà di Bruno Hopf algebra, the noncommutative version of the charge renormalization Hopf algebra on planar binary trees for quantum electrodynamics, and the non-commutative version of the Pinter renormalization Hopf algebra on any bosonic field.

We also describe two general ways to define the associative product in such Hopf algebras, the first one by recursion, and the second one by grafting and shuffling some decorated rooted trees.
\end{abstract}

Keywords Hopf algebra $\cdot$ Combinatorial $\cdot$ Brace $\cdot$ Tree $\cdot$ Renormalization

\section{Introduction}

In the paper [13], Loday and Ronco gave the definition of a right-sided combinatorial Hopf algebra (CHA) which includes the examples of Hopf algebras describing the renormalization in quantum field theory. The toy example, which inspired the work

C. Brouder

Institut de Minéralogie et de Physique des Milieux Condensés, CNRS UMR7590,

Universités Paris 6 et 7, IPGP, 140 rue de Lourmel, 75015 Paris, France

e-mail: christian.brouder@impmc.upmc.fr

\section{A. Frabetti (凶)}

Université de Lyon, Université Lyon 1, CNRS, UMR 5208 Institut Camille Jordan, 43, blvd du 11 novembre 1918, 69622 Villeurbanne, France

e-mail: frabetti@math.univ-lyon1.fr

F. Menous

Département de Mathématiques, Bât. 425, Université Paris-Sud, 91405 Orsay, France

e-mail: Frederic.Menous@math.u-psud.fr 
in [13], is Kreimer's Hopf algebra on rooted trees introduced in [9]. In this paper, we describe the CHA structure of three other such examples: the non-commutative version of the Faà di Bruno Hopf algebra given in [3], the non-commutative version of the charge renormalization Hopf algebra on planar binary trees given in [1] for quantum electrodynamics, and the non-commutative version of the Pinter renormalization Hopf algebra on bosonic quantum fields, as given in [4].

Since Loday and Ronco work in the context of cofree-coassociative CHAs, we describe our examples, which are all free-associative CHAs, by considering their linear duals. A right-sided cofree-coassociative CHA is completely determined by the brace structure on the set of its primitive elements. Therefore, to describe our examples, it will be sufficient to give their brace structure.

In the first section we fix the notation on right-sided cofree-coassociative CHAs and brace algebras from [13]. We also give a recursive definition of the product and show how to construct it starting from the one defined on decorated rooted trees, that is, the product defined on the right-sided CHA induced by the free brace algebra over the space of decorations. Then we fix the notation for the dual Hopf algebras, which are used in the sequel. Each example is then treated in a separate section.

\section{Combinatorial Hopf algebras from dual Hopf algebras}

\subsection{Right-sided combinatorial Hopf algebras}

In this section we recall the definition and main properties of a right-sided combinatorial Hopf algebra (r-s CHA), as given by Loday and Ronco in [13], in the cofreecoassociative case.

A right-sided cofree-coassociative $\mathrm{CHA}$ is, up to an isomorphism, a tensor coalgebra $T(R)=\bigoplus_{n \geq 0} R^{\otimes n}$ with the deconcatenation coproduct, endowed with an associative product $\star$ which makes it into a bialgebra and which satisfies the right-sided condition: for any $p \geq 0$, the subspace $T^{\geq p}(R)=\bigoplus_{n \geq p} R^{\otimes n}$ is a right ideal of $T(R)$, that is

$$
T^{\geq p}(R) \star T(R) \subset T^{\geq p}(R) .
$$

This condition is equivalent to demanding that the product $\star$ induces a right action of $T(R)$ on the quotient space $T_{<p}(R)=T(R) / T^{\geq p}(R)$, that is

$$
T_{<p}(R) \star T(R) \subset T_{<p}(R) .
$$

Loday and Ronco proved that a cofree-coassociative CHA is right-sided if and only if the set of its primitive elements $R$ is a brace algebra, that is, it is endowed with a brace product $\{; \ldots$,$\} acting on R \otimes T(R)$ with value in $R$, satisfying the brace relation

$$
\begin{aligned}
& \left\{\left\{x ; y_{1}, \ldots, y_{n}\right\} ; z_{1}, \ldots, z_{m}\right\} \\
& \quad=\sum\left\{x ; z_{1}, \ldots,\left\{y_{1} ; z_{k_{1}}, \ldots,\right\}, \ldots,\left\{y_{n} ; z_{k_{n}}, \ldots\right\}, \ldots, z_{m}\right\},
\end{aligned}
$$


for any $x, y_{i}, z_{j} \in R$. The brace product restricted to $R \otimes R^{\otimes q}$ is also denoted by $M_{1 q}$, if one wishes to specify the number of variables on the right, or to underline that it is a special case of a multibrace product $M_{p q}$ defined on any power $R^{\otimes p} \otimes R^{\otimes q}$.

The brace product can be found from the associative product $\star$ by projecting the result onto the space $R$ of co-generators. If we denote by $\pi: T(R) \rightarrow R$ the canonical projection, this means that

$$
M_{1 q}\left(x ; y_{1} \cdots y_{q}\right)=\pi\left(x \star\left(y_{1} \cdots y_{q}\right)\right),
$$

for any $x \in R$ and $y_{1} \cdots y_{q} \in R^{\otimes q}$, where we denote the tensor product in $T(R)$ by the concatenation. In particular, for any $x \in R$ we have

$$
M_{10}(x ; 1) \equiv M_{10}(x)=\pi(x \star 1)=x,
$$

therefore $M_{10}=$ Id on $R$.

Conversely, the product $\star$ in $T(R)$ can be reconstructed from the brace product on $R$, using the deconcatenation coproduct to recover $T(R)$ from $R$. The resulting formula of Loday and Ronco for the product is (cf. [13])

$$
\begin{aligned}
& \left(x_{1} \cdots x_{n}\right) \star\left(y_{1} \cdots y_{m}\right) \\
& \quad=\sum_{k \geq 1} \sum_{\begin{array}{c}
p_{1}+\cdots+p_{k}=n \\
p_{1}, \ldots, p_{k}=0,1
\end{array}} \sum_{\substack{q_{1}+\cdots+q_{k}=m \\
q_{1}, \ldots, q_{k} \geq 0}}\left[M_{p_{1} q_{1}} \cdots M_{p_{k} q_{k}}\right]\left(x_{1} \cdots x_{n} ; y_{1} \cdots y_{m}\right),
\end{aligned}
$$

where each map $M_{p q}$ is applied to a block of $p$ generators $x$ and $q$ generators $y$, with the following assumptions:

$$
\begin{aligned}
& M_{00}=0 \quad \text { and } \quad M_{01}=M_{10}=\mathrm{Id}, \\
& M_{0 p}=M_{p 0}=0 \quad \text { for } p>1, \\
& M_{p q}=0 \quad \text { for } p>1 \text { and } q \neq 0,
\end{aligned}
$$

and where the operators $M_{1 q}$ for $q \neq 0$ of course satisfy the brace relation (2.2).

Applying formula (2.3) requires some computations. Let us take the example of $x \star y$. For $k=1$ we have $p_{1}=q_{1}=1$ and the only term is $M_{11}$. For $k=2$ we have $\left(p_{1}, p_{2}\right)=(1,0)$ or $\left(p_{1}, p_{2}\right)=(0,1)$ and $\left(q_{1}, q_{2}\right)=(1,0)$ or $\left(q_{1}, q_{2}\right)=(0,1)$. This gives us the four terms $M_{11} M_{00}+M_{10} M_{01}+M_{01} M_{10}+M_{00} M_{11}$. Since $M_{00}=0$, we have only two terms left $M_{10} M_{01}+M_{01} M_{10}$. For $k>2$, all the terms are zero because each term contains a factor $M_{00}$. In general, the terms with $k>n+m$ are zero in the product $\left(x_{1} \cdots x_{n}\right) \star\left(y_{1} \cdots y_{m}\right)$. Therefore, using (2.4), we get

$$
\begin{aligned}
x \star y & =M_{11}(x ; y)+\left[M_{10} M_{01}\right](x ; y)+\left[M_{01} M_{10}\right](x ; y) \\
& =M_{11}(x ; y)+M_{10}(x) M_{01}(y)+M_{01}(y) M_{10}(x)=M_{11}(x ; y)+x y+y x .
\end{aligned}
$$

Similarly, for $(x y) \star z$, we have two terms for $k=2: M_{11} M_{10}+M_{10} M_{11}$ and three terms for $k=3: M_{01} M_{10} M_{10}+M_{10} M_{01} M_{10}+M_{10} M_{10} M_{01}$, so that

$$
(x y) \star z=x M_{11}(y ; z)+M_{11}(x ; z) y+z x y+x z y+x y z .
$$


For $x \star(y z)$, we have one term for $k=1: M_{12}$, two terms for $k=2: M_{11} M_{01}+$ $M_{01} M_{11}$ and three terms for $k=3: M_{01} M_{01} M_{10}+M_{01} M_{10} M_{01}+M_{10} M_{01} M_{01}$, so that

$$
x \star(y z)=M_{12}(x ; y z)+y M_{11}(x ; z)+M_{11}(x ; y) z+y z x+y x z+x y z .
$$

In particular then, formula (2.3) for $n=1$ gives

$$
x \star\left(y_{1} \cdots y_{m}\right)=\sum_{i=0}^{m} \sum_{q=0}^{m-i} y_{1} \cdots y_{i} M_{1 q}\left(x ; y_{i+1} \cdots y_{i+q}\right) y_{i+q+1} \cdots y_{m} .
$$

2.2 Recursive definition of the product

Formula (2.3) can be given in a recursive way. To do it, let us denote by $M: R \otimes$ $T(R) \rightarrow R$ the brace product $\{; \ldots$,$\} induced on R$ by the associative product $\star$ on $T(R)$, and let us denote by $X, Y, Z, \ldots$ the generic words in $T(R)$.

Proposition 2.1 Given the brace product $M$ on $R$, the $\star$ product on $T(R)$ can be reconstructed in the following recursive way. For any $X, Y \in T(R)$, with $X, Y \notin T^{0}(R)$ :

$$
\begin{aligned}
X \star Y & =\sum_{\substack{X=X^{1} X^{2} \\
Y=Y^{1} Y^{2}}}\left(X^{1} \star Y^{1}\right) M\left(X^{2} ; Y^{2}\right) \\
& =\sum_{\substack{X=X^{1} X^{2} \\
Y=Y^{1} Y^{2}}} M\left(X^{1} ; Y^{1}\right)\left(X^{2} \star Y^{2}\right) \\
& =\sum_{\substack{X=X^{1} X^{2} X^{3} \\
Y=Y^{1} Y^{2} Y^{3}}}\left(X^{1} \star Y^{1}\right) M\left(X^{2}, Y^{2}\right)\left(X^{3} \star Y^{3}\right),
\end{aligned}
$$

where the sums run over all possible factorizations of $X$ and $Y$ with respect to the concatenation.

Proof With the assumptions (2.4) on $M$, formula (2.3) can be expressed as

$$
X \star Y=\sum_{k \geq 1} \sum_{\substack{X=X^{1} \ldots X^{k} \\ Y=Y^{1} \ldots Y^{k}}} M\left(X^{1} ; Y^{1}\right) \cdots M\left(X^{k} ; Y^{k}\right)
$$

where the sum runs other all the factorizations (for the concatenation) of $X=$ $X^{1} \cdots X^{k}$ and $Y=Y^{1} \cdots Y^{k}$. Note that the factors can be in $T^{0}(R)$ but the condition $M(1 ; 1)=0$ ensures that the sum is finite. It also ensures that formula (2.6) is recursive.

If we then use this formula to express the factor $X^{1} \star Y^{1}$ in line (2.6), we obtain the desired expression for $X \star Y$. 
In particular, if we expand the words $X$ and $Y$ of $T(R)$ in terms of elements $x_{i}$ and $y_{j}$ of $R$, with the notation of Sect. 2.1, formula (2.6) becomes

$$
\begin{aligned}
& \left(x_{1} \cdots x_{n}\right) \star\left(y_{1} \cdots y_{m}\right) \\
& =\left[\left(x_{1} \cdots x_{n-1}\right) \star\left(y_{1} \cdots y_{m}\right)\right] x_{n}+\left[\left(x_{1} \cdots x_{n}\right) \star\left(y_{1} \cdots y_{m-1}\right)\right] y_{m} \\
& \quad+\sum_{i=1}^{m-1}\left[\left(x_{1} \cdots x_{n-1}\right) \star\left(y_{1} \cdots y_{i}\right)\right] M_{1 m-i}\left(x_{n} ; y_{i+1} \cdots y_{m}\right) .
\end{aligned}
$$

Corollary 2.2 The brace relation (2.2) is equivalent to the identity

$$
M(M(X ; Y) ; Z)=M(X ; Y \star Z),
$$

for any $X \in R$ and $Y, Z \in T(R)$.

Proof If we call $X=x, Y=y_{1} \cdots y_{n}$ and $Z=z_{1} \cdots z_{m}$, the left-hand side of the brace relation (2.2) is simply $M(M(X ; Y) ; Z)$. The right-hand side, instead, becomes

$$
\sum_{k \geq 1} \sum_{\substack{Y=Y^{1} \ldots Y^{k} \\ Z=Z^{1} \cdots Z^{k}}} M\left(X ; M\left(Y^{1} ; Z^{1}\right) \cdots M\left(Y^{k} ; Z^{k}\right)\right),
$$

where we use the assumptions (2.4) on $M$. From (2.7) it then follows that the righthand side is equal to $M(X ; Y \star Z)$.

Remark 2.3 The right-sided combinatorial Hopf algebras, as well as the shuffle and the quasishuffle Hopf algebras, are examples of more general cofree-coassociative combinatorial Hopf algebras $T(R)$ introduced by Loday and Ronco, where $R$ is a so-called multibrace algebra. See [13] and the references therein for details. In all these examples, as well as in the general case, the product $\star$ on $T(R)$ can be defined recursively from the multibrace product on $R$. The expressions (2.7), (2.6) and (2.9), with $X \in T(R)$, are valid in the most general case as well as in all the examples.

\subsection{Right-sided Hopf algebras and trees}

Let $T(R)$ be a right-sided CHA such that the brace algebra $R$ is a finite dimensional or a graded vector space. In the first part of this section we forget the brace structure on $R$ and consider the free brace algebra on $R$ modeled by planar rooted trees. The free brace algebra has already been described in terms of trees by Chapoton in [5], as a consequence of his construction of the Brace operad. Here we give a direct description of the free brace algebra within the notations we established in Sect. 2.2.

In the second part of this section we relate the free brace structure on $R$ with the original one, and deduce a way to describe the associative product $\star$ on $T(R)$ starting from the one defined on trees. Again, this is a particular case of the equivalence 
between the categories of brace and dendriform algebras given by Chapoton in [5]. However, it seems to us useful for computations to write an explicit statement.

Let $\mathcal{T}$ denote the set of plane rooted trees and for any non-empty tree $t$ denote by $V(t)$ the set of its vertices and by $|t|$ the cardinality of $V(t)$. If $d$ is a map from $V(t)$ to $R^{\otimes|t|}$, then the pair $(t, d)$ is a plane tree with decorations in $R$. For example, the following tree with 4 vertices is decorated by the given map $d$ :

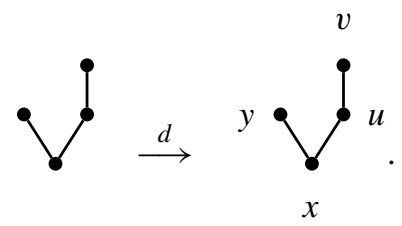

For any $t \in \mathcal{T}$, we denote by $R^{t}$ the linear span of all the decorated trees of shape $t$, modulo the obvious linear relations coming from $R$, such as:

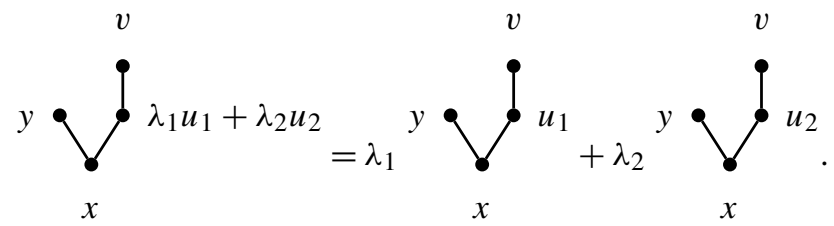

Then the vector space

$$
R^{\mathcal{T}}=\bigoplus_{t \in \mathcal{T}} R^{t}
$$

contains the decorated trees of any shape, and the elements of the tensor space $T\left(R^{\mathcal{T}}\right)$ are called decorated forests. From now on, we denote by the concatenation the tensor product between trees, and we simply denote by $t$ a decorated tree, omitting the decoration.

We now define the structure of a brace algebra on $R^{\mathcal{T}}$, and consequently that of a right-sided CHA on $T\left(R^{\mathcal{T}}\right)$, using the results of Sect. 2.2. Therefore, we endow $T\left(R^{\mathcal{T}}\right)$ with the free (deconcatenation) coproduct and we suppose that the product $\star$ is defined through the brace product $M$ on $R^{\mathcal{T}}$.

To define the brace product, consider, for any $x \in R$, the linear map

$$
B_{+}^{x}: T\left(R^{\mathcal{T}}\right) \rightarrow R^{\mathcal{T}}
$$

which maps a forest $f=t_{1} \cdots t_{n}$ into the tree $t=B_{+}^{x}(f)$ obtained by joining the ordered trees $t_{1}, \ldots, t_{n}$ to a new root decorated by $x$. For instance

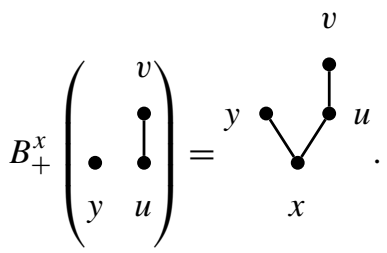


Any tree $t$ can be written as $B_{+}^{x}(f)$. If $t$ is made only of the root, decorated by $x$, the forest $f$ is the formal unit of $T\left(R^{\mathcal{T}}\right)$, that is $1 \in\left(R^{\mathcal{T}}\right)^{0}$.

Theorem 2.4 Let $M: R^{\mathcal{T}} \otimes T\left(R^{\mathcal{T}}\right) \rightarrow R^{\mathcal{T}}$ be the linear map defined on any decorated tree $t=B_{+}^{x}\left(f_{1}\right)$ and any decorated forest $f_{2}$ by

$$
M\left(B_{+}^{x}\left(f_{1}\right) ; f_{2}\right)=B_{+}^{x}\left(f_{1} \star f_{2}\right),
$$

where $\star$ is the product induced by $M$ on $T\left(R^{\mathcal{T}}\right)$ according to $(2.7)$ and with the assumptions (2.4).

Then $M$ is a brace product on $R^{\mathcal{T}}$, and consequently $\star$ is an associative product on $T\left(R^{\mathcal{T}}\right)$ which makes it into a right-sided $C H A$.

Proof Assuming conditions (2.4) on the maps $M_{p q}$, it suffices to show that $M$ satisfies the brace identity (2.9), that is

$$
M\left(M\left(t_{1} ; f_{2}\right) ; f_{3}\right)=M\left(t_{1} ; f_{2} \star f_{3}\right)
$$

for any tree $t_{1}$ and any forests $f_{2}, f_{3}$. If $t_{1}, f_{2}, f_{3}$ have respectively $n_{1}, n_{2}$ and $n_{3}$ vertices, we prove the brace identity by induction on the total number of vertices $n_{1}+n_{2}+n_{3}$.

Since $t_{1}$ is a tree, it has at least one vertex (the root) and therefore $n_{1}+n_{2}+n_{3} \geq 1$. If $n_{1}+n_{2}+n_{3}=1$, only $t_{1}$ is a tree, namely the single root, and $f_{2}, f_{3}$ are scalars. Because $1 \star 1=1$ and $M_{10}=M_{01}=\mathrm{Id}$, both terms in the equality produce $t_{1}$ and therefore coincide.

Now suppose that the brace identity holds for $n_{1}+n_{2}+n_{3} \leq n$, for a given $n \geq 1$. According to the previous sections, this implies that the product $\star$ is associative up to the same number $n$ of vertices. (In fact, the associativity of $\star$ does not depend on the definition (2.10) of $M$ and was proved in general by Loday and Ronco.) Then consider a tree and two forests with a total number of vertices $n_{1}+n_{2}+n_{3}=n+1$. Using the definition of $M$ and the associativity of $\star$ up to $n$, for $t_{1}=B_{+}^{x}\left(f_{1}\right)$ we obtain

$$
\begin{aligned}
M\left(M\left(B_{+}^{x}\left(f_{1}\right) ; f_{2}\right) ; f_{3}\right) & =M\left(B_{+}^{x}\left(f_{1} \star f_{2}\right) ; f_{3}\right) \\
& =B_{+}^{x}\left(\left(f_{1} \star f_{2}\right) \star f_{3}\right) \quad(\text { Recursion }) \\
& =B_{+}^{x}\left(f_{1} \star\left(f_{2} \star f_{3}\right)\right) \\
& =M\left(B_{+}^{x}\left(f_{1}\right) ; f_{2} \star f_{3}\right) .
\end{aligned}
$$

Lemma 2.5 The brace product $M$ on $R^{\mathcal{T}}$ can be expressed in terms of graftings of trees: if $t \in R^{\mathcal{T}}$ and $s_{1} \cdots s_{n} \in T\left(R^{\mathcal{T}}\right)$, then $M\left(t ; s_{1} \cdots s_{n}\right)$ is the sum of the terms obtained by grafting the trees $s_{1}, \ldots, s_{n}$ onto the vertices of $t$, in all the possible ways which preserve the order of the $s_{i}$ 's from left to right.

Similarly, the product $\star$ on $T\left(R^{\mathcal{T}}\right)$ is the sum of terms mixing all possible shuffles and all possible graftings of the trees on the right-hand side onto the vertices of the trees on the left-hand side. 
This result shows that the recursive definition of the brace product $(2.10)$ is equivalent to the definition given in [5] in terms of grafting of trees.

Proof We prove it by induction on the size of the forests, given by the total number of vertices. For this, we need to treat at the same time $M$ and $\star$, which is given by (2.6).

The induction starts with the first non-trivial operations:

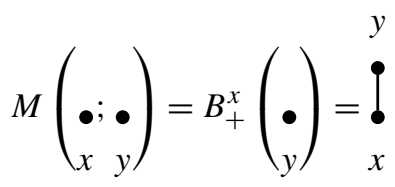

is the grafting of the second $\bullet$ on the first one. The claim is obtained because this is the only possible grafting. Similarly, according to (2.6), the product

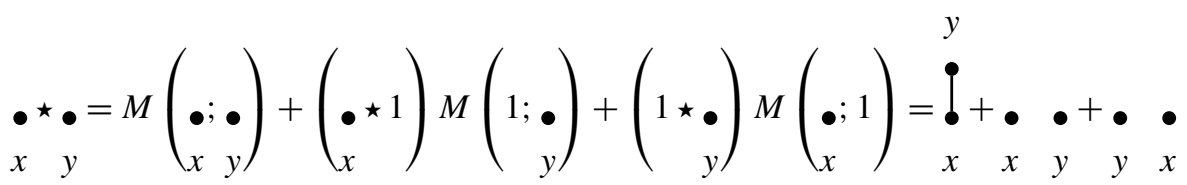

is exactly made of the only possible grafting and the two allowed shuffles.

Now suppose that $M\left(t_{1} ; f_{2}\right)$ is made of all possible ordered graftings of the trees composing the forest $f_{2}$ on the vertices of the tree $t_{1}$, for $t_{1}$ and $f_{2}$ having a total number of vertices $n_{1}+n_{2} \leq n$, for some $n \geq 1$. Similarly, suppose that $f_{1} \star f_{2}$ is made of the terms mixing all possible shuffles of the trees of $f_{1}$ with those of $f_{2}$, and of graftings of the trees of $f_{2}$ over the vertices of those contained in $f_{1}$, for $f_{1}$ and $f_{2}$ having a total number of vertices $n_{1}+n_{2} \leq n$.

Then, consider $t_{1}$ and $f_{2}$ with $n_{1}+n_{2}=n+1$. Applying the definition (2.10) for $t_{1}=B_{+}^{x}\left(f_{1}\right)$, we see that

$$
M\left(B_{+}^{x}\left(f_{1}\right) ; f_{2}\right)=B_{+}^{x}\left(f_{1} \star f_{2}\right)
$$

is the grafting of $f_{1} \star f_{2}$ onto the original root. The claim is then guaranteed by the inductive hypothesis on $f_{1} \star f_{2}$.

Similarly, consider $t_{1}$ and $f_{2}$ with $n_{1}+n_{2}=n+1$. Applying formula (2.6) we see that

$$
f_{1} \star f_{2}=\sum_{\substack{f_{1}=f_{1}^{\prime} f_{2}^{\prime \prime} \\ f_{2}=f_{1}^{\prime} f_{2}^{\prime \prime}}}\left(f_{1}^{\prime} \star f_{2}^{\prime}\right) M\left(f_{1}^{\prime \prime} ; f_{2}^{\prime \prime}\right)
$$

contains exactly all possible shuffles mixed with all possible graftings. This is guaranteed by the inductive hypothesis on the factors $f_{1}^{\prime} \star f_{2}^{\prime}$ and $M\left(f_{1}^{\prime \prime} ; f_{2}^{\prime \prime}\right)$, and the fact that the sum runs over all decompositions of $f_{1}$ and $f_{2}$ into two factors, including the trivial ones.

Here are some examples of graftings and shuffles: 


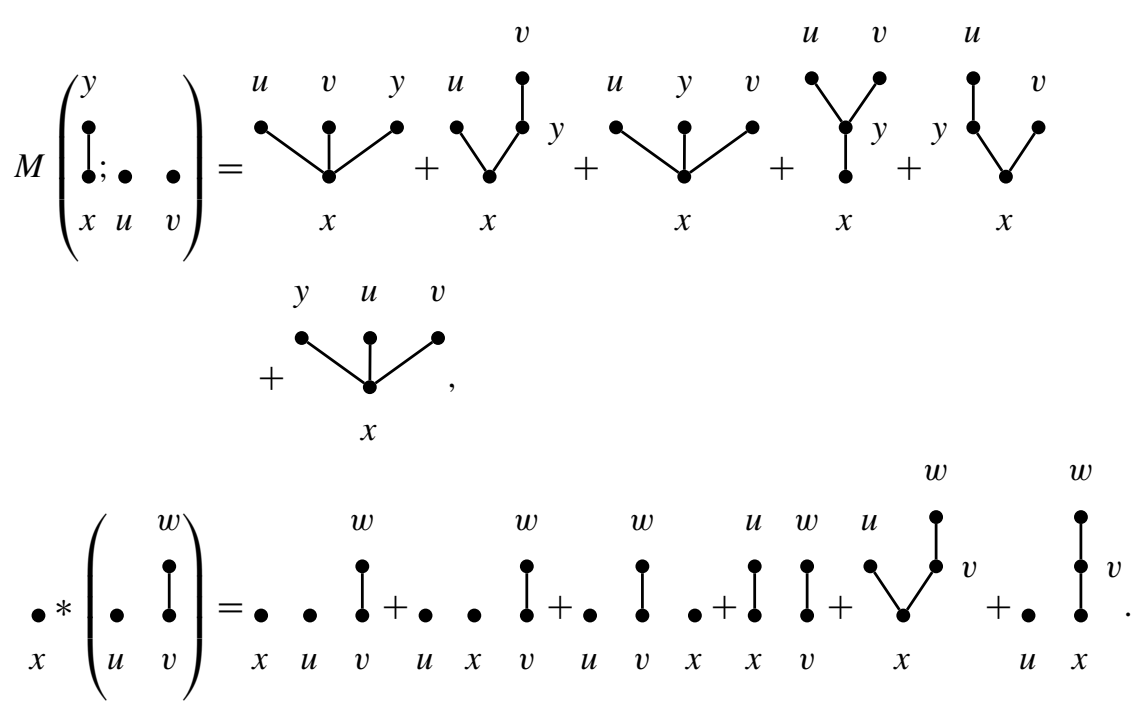

According to the results of Loday and Ronco, in particular paragraph 3.14 in [13], we can conclude that

Corollary 2.6 The brace algebra $R^{\mathcal{T}}$, endowed with $M$, is the free brace algebra over $R$.

Remark 2.7 The algebras $R^{\mathcal{T}}$ and $T\left(R^{\mathcal{T}}\right)$, or their duals, or their non-planar and commutative versions, appeared in many recent works. We quote, but the list is surely not exhaustive, the works by Grossman and Larson [7], Chapoton [5], Foissy [6], Guin and Oudom [8], Ronco [15] and Loday and Ronco [13].

Now suppose that $R$ itself is a brace algebra, and therefore $T(R)$ is a right-sided CHA. We are going to show that the $\star$ product defined in $T(R)$ can be found by computing its lifting to $T\left(R^{\mathcal{T}}\right)$.

Let $\iota$ be the linear morphism from $T(R)$ to $T\left(R^{\mathcal{T}}\right)$ defined by $\iota(1)=1$ and

$$
\iota\left(x_{1} \cdots x_{n}\right)=B_{+}^{x_{1}}(1) \cdots B_{+}^{x_{n}}(1)=\begin{array}{cc}
\bullet & \bullet \\
x_{1} & x_{n}
\end{array}
$$

It is clear that $\iota$ preserves the concatenation in $T(R)$ and $T\left(R^{\mathcal{T}}\right)$, and that it identifies $R$ with $R^{\bullet} \subset R^{\mathcal{T}}$.

Note that the map $\iota$ does not preserve the brace product, in fact

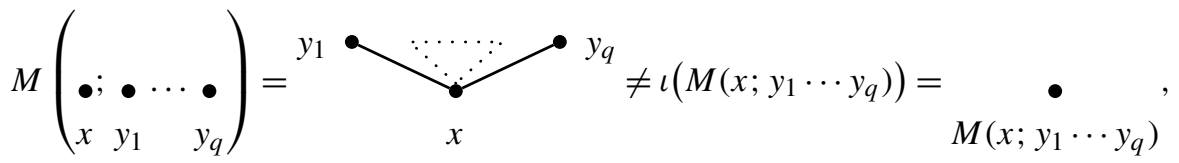

and therefore it does not preserve the product $\star$ in $T(R)$ and $T\left(R^{\mathcal{T}}\right)$. We show that $\iota$ has an inverse map which allows to compute the product $\star$ in $T(R)$ starting from that in $T\left(R^{\mathcal{T}}\right)$. 
Theorem 2.8 Let $\mu$ the linear morphism from $T\left(R^{\mathcal{T}}\right)$ to $T(R)$ defined recursively by

1. $\mu(1)=1$,

2. $\mu\left(t_{1} \ldots t_{n}\right)=\mu\left(t_{1}\right) \ldots \mu\left(t_{n}\right)$,

3. $\mu\left(B_{+}^{x}\left(s_{1} \ldots s_{q}\right)\right)=M_{1 q}\left(x ; \mu\left(s_{1} \ldots s_{q}\right)\right)$.

Then, for any $X, Y \in T(R)$, we have

$$
X \star Y=\mu(\iota(X) \star \iota(Y)) .
$$

Proof The proof is straightforward and relies on the recursive formulas in brace algebras and on the fact that $\mu \circ \iota=\operatorname{Id}_{T(R)}$. If $X=x_{1} \cdots x_{p}$ and $Y=y_{1} \cdots y_{q}$ then

$$
X \star Y=\sum_{\substack{X=X^{1} X^{2} \\ Y=Y^{1} Y^{2}}}\left(X^{1} \star Y^{1}\right) M\left(X^{2} ; Y^{2}\right)
$$

Since $R$ is a brace algebra, either $X^{2}=1$ or $X^{2}=x_{p}$.

- If $X^{2}=1$, then

$$
M\left(1, Y^{2}\right)=Y^{2}=\mu\left(\iota\left(Y^{2}\right)\right)=\mu\left(M\left(1 ; \iota\left(Y^{2}\right)\right)\right) .
$$

- If $X^{2}=x_{p}$, then

$$
M\left(x_{p}, Y^{2}\right)=\mu\left(B_{+}^{x_{p}}\left(\iota\left(Y^{2}\right)\right)\right)=\mu\left(M\left(\iota\left(x_{p}\right) ; \iota\left(Y^{2}\right)\right)\right) .
$$

Recursively, we get

$$
\begin{aligned}
X \star Y & =\sum_{\substack{X=X^{1} X^{2} \\
Y=Y^{1} Y^{2}}}\left(X^{1} \star Y^{1}\right) M\left(X^{2} ; Y^{2}\right) \\
& =\sum_{\substack{X=X^{1} X^{2} \\
Y=Y^{1} Y^{2}}} \mu\left(\iota\left(X^{1}\right) \star \iota\left(Y^{1}\right)\right) \mu\left(M\left(\iota\left(X^{2}\right) ; \iota\left(Y^{2}\right)\right)\right) \\
& =\mu\left(\sum_{\substack{\iota(X)=\iota\left(X^{1}\right) \iota\left(X^{2}\right) \\
\iota(Y)=\iota\left(Y^{1}\right) \iota\left(Y^{2}\right)}}\left(\iota\left(X^{1}\right) \star \iota\left(Y^{1}\right)\right) M\left(\iota\left(X^{2}\right) ; \iota\left(Y^{2}\right)\right)\right) \\
& =\mu(\iota(X) \star \iota(Y)) .
\end{aligned}
$$

This result means that one can compute the $\star$ product in $T(R)$ with the help of the $\star$ product in $T\left(R^{\mathcal{T}}\right)$. This last product, given by shuffle and grafting of trees, is quite easy to handle. Let us illustrate the application with an example: 


$$
\begin{aligned}
& x \star(y z)=\mu\left(\bullet \star\left(\begin{array}{ll}
\bullet & \bullet \\
y & z
\end{array}\right)\right)
\end{aligned}
$$

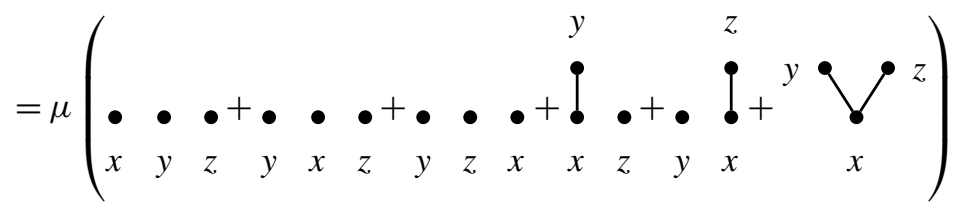

$$
\begin{aligned}
& =x y z+y x z+y z x+M_{11}(x ; y) z+y M_{11}(x ; z)+M_{12}(x ; y z) \text {. }
\end{aligned}
$$

This computation can be compared with that made in Sect. 2.1.

Remark 2.9 The result of theorem (2.8) can be obtained also as a consequence of the equivalence between the categories of brace and dendriform algebras proved by Chapoton in [5]. Since $\mu$ is by assumption a brace homomorphism between $R^{\mathcal{T}}$ and $R$, this equivalence allows to state that for any $f_{1}$ and $f_{2}$ in $T\left(R^{\mathcal{T}}\right)$ we have $\mu\left(f_{1}\right) \star \mu\left(f_{2}\right)=\mu\left(f_{1} \star f_{2}\right)$. If we then choose $f_{1}=\iota(X)$ and $f_{2}=\iota(Y)$, we obtain formula (2.11).

Remark 2.10 The algebra $T\left(R^{\mathcal{T}}\right)$ is the dual of Foissy's Hopf algebra of decorated plane trees, cf. [6]. Foissy's Hopf algebra on rooted trees has a "universal property", in the sense that it is endowed with the Hochschild cocycle operator $B_{+}$. Note that the property that we show for $T\left(R^{\mathcal{T}}\right)$ is not the dual property of that one.

\subsection{Right-sided CHAs from dual Hopf algebras}

In the next sections we determine some right-sided CHAs $T(R)$ from the dualization of given Hopf algebras $T(V)$ where the vector space $V$ is itself graded. In this case, the vector space $R$ coincides with the dual space $V^{*}$, and we give a general tool to compute the brace structure on $R$ starting from the coproduct on $T(V)$. This tool is then used in the examples presented in the rest of the paper.

Let $\mathcal{H}$ be a graded Hopf algebra that is free as an algebra, finite dimensional in all degrees, and with generators which are themselves graded. If we denote by $V$ be the vector space spanned by all the generators of $\mathcal{H}$ (infinite but countable many), the Hopf algebra can be given as $\mathcal{H}=T(V)=\bigoplus_{n=0}^{\infty} V^{\otimes n}$.

The dual Hopf algebra $\mathcal{H}^{*}$ is defined as follows. As a vector space, it is the graded linear dual vector space of $\mathcal{H}$, that is $\mathcal{H}^{*}=T\left(V^{*}\right)$, where $V^{*}$ is the graded linear dual vector space of $V$. If we denote by $\left\{v_{n}, n \geq 1\right\}$ a generic basis of $V$, that is a set of generators of $\mathcal{H}$, the element $v_{n}^{*}$ dual to each $v_{n}$ is defined by the pairing $\left\langle v_{n}^{*} \mid v_{m}\right\rangle=\delta_{n, m}$. Therefore, the dual of a generic basis element $v_{n_{1}} \cdots v_{n_{i}}$ in $\mathcal{H}$ is the element $v_{n_{1}}^{*} \cdots v_{n_{i}}^{*}$ in $\mathcal{H}^{*}$ defined by the pairing

$$
\left\langle v_{n_{1}}^{*} \cdots v_{n_{i}}^{*} \mid v_{m_{1}} \cdots v_{m_{j}}\right\rangle=\delta_{i, j}\left\langle v_{n_{1}}^{*} \mid v_{m_{1}}\right\rangle \ldots\left\langle v_{n_{i}}^{*} \mid v_{m_{i}}\right\rangle .
$$

As a coalgebra, $\mathcal{H}^{*}$ inherits the coproduct $\Delta^{*}$ dual to the product of $\mathcal{H}$, that is, such that

$$
\left\langle\Delta^{*}\left(v_{n_{1}}^{*} \cdots v_{n_{i}}^{*}\right) \mid\left(v_{m_{1}} \cdots v_{m_{j}}\right) \otimes\left(v_{p_{1}} \cdots v_{p_{k}}\right)\right\rangle=\left\langle v_{n_{1}}^{*} \cdots v_{n_{i}}^{*} \mid v_{m_{1}} \cdots v_{m_{j}} v_{p_{1}} \cdots v_{p_{k}}\right\rangle .
$$


Since $\mathcal{H}$ is free and unital as an algebra, $\mathcal{H}^{*}$ is cofree and counital as a coalgebra, that is, $\Delta^{*}$ is the deconcatenation coproduct

$$
\begin{aligned}
\Delta^{*}\left(v_{n}^{*}\right) & =v_{n}^{*} \otimes 1+1 \otimes v_{n}^{*}, \\
\Delta^{*}\left(v_{n_{1}}^{*} \cdots v_{n_{k}}^{*}\right) & =\sum_{i=0}^{k} v_{n_{1}}^{*} \cdots v_{n_{i}}^{*} \otimes v_{n_{i+1}}^{*} \cdots v_{n_{k}}^{*},
\end{aligned}
$$

and with the counit $\varepsilon=1^{*}$. In this expression, 1 is not (yet) a unit, it denotes the generator of $\left(V^{*}\right)^{\otimes 0}$. The set of primitive elements of $\mathcal{H}^{*}$ is then obviously the dual $V^{*}$ of the set of generators of $\mathcal{H}$.

Finally, as an algebra, $\mathcal{H}^{*}$ inherits the product $\star$ dual to the coproduct $\Delta$ of $\mathcal{H}$, that is, the product such that

$\left\langle\left(v_{n_{1}}^{*} \cdots v_{n_{i}}^{*}\right) \star\left(v_{m_{1}}^{*} \cdots v_{m_{j}}^{*}\right), v_{p_{1}} \cdots v_{p_{k}}\right\rangle=\left\langle v_{n_{1}}^{*} \cdots v_{n_{i}}^{*} \otimes v_{m_{1}}^{*} \cdots v_{m_{j}}^{*}, \Delta\left(v_{p_{1}} \cdots v_{p_{k}}\right)\right\rangle$.

The right-sided condition (2.1) for the Hopf algebra $T\left(V^{*}\right)$ is equivalent to the requirement that the coproduct $\Delta$ on $T(V)$ induces a right coaction of the Hopf algebra $T(V)$ on the subspace $T \leq p(V)=\bigoplus_{n \leq p} V^{\otimes n}$, that is

$$
\Delta\left(T^{\leq p}(V)\right) \subset T^{\leq p}(V) \otimes T(V)
$$

Explicitly, this means that for any element $a=v_{n_{1}} \cdots v_{n_{p}} \in V^{\otimes p}$, the coproduct $\Delta(a)=\sum a_{(1)} \otimes a_{(2)}$ (in Sweedler's notation) produces terms with $a_{(1)} \in T \leq p(V)$. Since the coproduct is an algebra morphism, and the product is free, it is sufficient that this property holds for $p=1$ : for any generator $v \in V$, the coproduct $\Delta(v)=\sum v_{(1)} \otimes v_{(2)}$ produces terms with $v_{(1)} \in T^{\leq 1}(V)=K \oplus V$ (cf. [13], Sect. 3.6).

Finally, the brace product on $V^{*}$ is the projection of $\star$ onto $V^{*}$. The projection $\pi: T\left(V^{*}\right) \rightarrow V^{*}$, in this case, is the map

$$
\pi\left(a^{*}\right)=\sum_{m}\left\langle a^{*} \mid v_{m}\right\rangle v_{m}^{*}
$$

for any $a^{*} \in T\left(V^{*}\right)$. Therefore, the maps $M_{1 q}: V^{*} \otimes\left(V^{*}\right)^{\otimes q} \rightarrow V^{*}$ are simply given by

$$
M_{1 q}\left(v_{n}^{*} ; a^{*}\right)=\sum_{m}\left\langle v_{n}^{*} \star a^{*} \mid v_{m}\right\rangle v_{m}^{*}=\sum_{m}\left\langle v_{n}^{*} \otimes a^{*} \mid \Delta v_{m}\right\rangle v_{m}^{*}
$$

This sum is infinite. In fact, let us denote by $|a|$ [resp. $\left.\left|a^{*}\right|\right]$ the degree of the elements in the graded Hopf algebra $T(V)$ [resp. $T\left(V^{*}\right)$ ]. We recall that if $\left|v_{n}\right|$ denotes the degree of a generic basis element of $V$, then the degree of an element $v_{n_{1}} \cdots v_{n_{p}} \in T(V)$ is given by $\left|v_{n_{1}} \cdots v_{n_{p}}\right|=\left|v_{n_{1}}\right|+\cdots+\left|v_{n_{p}}\right|$. Then the sum over $m$ in (2.13) is limited by the fact that $\left|v_{m}^{*}\right|=\left|v_{m}\right|=\left|v_{n}^{*}\right|+\left|a^{*}\right|$. 


\section{The right-sided combinatorial structure of the Hopf algebra $\left(\mathcal{H}^{\text {dif,nc }}\right)^{*}$}

Let $\mathcal{H}^{\text {dif,nc }}$ be the non-commutative Hopf algebra of formal diffeomorphisms, as defined in [16] or [3] (where it was denoted by $\mathcal{H}^{\text {dif }}$ ). It is the graded and connected Hopf algebra $\mathbb{Q}\left\langle v_{1}, v_{2}, \ldots\right\rangle=T(V)$ on the generators $V=\operatorname{Span}\left\{v_{1}, v_{2}, \ldots\right\}$ graded by $\left|v_{n}\right|=n$, considered with the free (tensor) product, and endowed with the coproduct

$$
\Delta^{\operatorname{dif}}\left(v_{n}\right)=\sum_{m=0}^{n} v_{m} \otimes \sum_{\begin{array}{c}
k_{0}, k_{1}, \ldots, k_{m} \geq 0 \\
k_{0}+k_{1}+\cdots+k_{m}=n-m
\end{array}} v_{k_{0}} v_{k_{1}} \cdots v_{k_{m}},
$$

where we set $v_{0}=1$, and with the counit $\varepsilon\left(v_{n}\right)=\delta_{n, 0}$. This Hopf algebra is a freeassociative CHA, and the formula (3.1) clearly says that the coproduct $\Delta^{\text {dif }}$ satisfies the right-sided condition (2.12).

Let $\left(\mathcal{H}^{\text {dif,nc }}\right)^{*}=T\left(V^{*}\right)$ be its dual Hopf algebra, as described in Sect. 2, with primitive elements in $V^{*}=\operatorname{Span}\left\{v_{1}^{*}, v_{2}^{*}, \ldots\right\}$. Then $\left(\mathcal{H}^{\text {dif,nc }}\right)^{*}=T\left(V^{*}\right)$ is a rightsided cofree-coassociative CHA, and moreover a dendriform algebra, and $V^{*}$ is a brace algebra.

Proposition 3.1 The brace product on $V^{*}$ which induces the CHA structure on $\left(\mathcal{H}^{\text {dif,nc }}\right)^{*}$ is given by

$$
\left\{v_{n}^{*} ; v_{m_{1}}^{*} \cdots v_{m_{q}}^{*}\right\}=M_{1 q}\left(v_{n}^{*} ; v_{m_{1}}^{*} \cdots v_{m_{q}}^{*}\right)=\left(\begin{array}{c}
n+1 \\
q
\end{array}\right) v_{n+m_{1}+\cdots+m_{q}}^{*} .
$$

Proof According to (2.13), for any $v_{n} \in V$ and any $a \in \mathcal{H}^{\text {dif,nc }}$, we have

$$
M_{1 q}\left(v_{n}^{*} ; a^{*}\right)=\sum_{m=1}^{\infty}\left\langle v_{n}^{*} \otimes a^{*} \mid \Delta^{\mathrm{dif}} v_{m}\right\rangle v_{m}^{*}
$$

If we put $v_{0}=1$, the coproduct of $v_{m}$ becomes [3]

$$
\Delta^{\mathrm{dif}} v_{m}=\sum_{k=0}^{m} v_{k} \otimes Q_{m-k}^{(k)}(v)
$$

with

$$
Q_{m-k}^{(k)}(v)=\sum_{l=1}^{k+1}\left(\begin{array}{c}
k+1 \\
l
\end{array}\right) \sum_{\substack{j_{1}, \ldots, j_{l}>0 \\
j_{1}+\cdots+j_{l}=m-k}} v_{j_{1}} \cdots v_{j_{l}}
$$

Therefore,

$$
M_{1 q}\left(v_{n}^{*} ; a^{*}\right)=\sum_{m=1}^{\infty}\left\langle a^{*}\left|Q_{m-n}^{(n)}(v)\right| v_{m}^{*}\right.
$$


We take $a=v_{m_{1}} \cdots v_{m_{q}}$ and we evaluate $\left\langle v_{m_{1}}^{*} \cdots v_{m_{q}}^{*} \mid Q_{m-n}^{(n)}(v)\right\rangle$. From the definition of $Q_{m-n}^{(n)}(v)$, we must have $l=q, j_{1}=m_{1}, \ldots, j_{q}=m_{q}$. Therefore, $m-n=j_{1}+$ $\cdots+j_{q}=m_{1}+\cdots+m_{q}$ and

$$
M_{1 q}\left(v_{n}^{*} ; v_{m_{1}}^{*} \cdots v_{m_{q}}^{*}\right)=\left(\begin{array}{c}
n+1 \\
q
\end{array}\right) v_{n+m_{1}+\cdots+m_{q}}^{*} .
$$

\section{The right-sided combinatorial structure of the Hopf algebra $\left(\mathcal{H}^{\alpha, n c}\right)^{*}$}

Let $Y$ be the set of planar binary rooted trees, that is, planar graphs without loops with internal vertices of valence three and a preferred external edge called the root. For instance,

$$
\text { |, Y, Y, Y, Y, Y, YY, Y, Y. }
$$

For a tree $t$, we denote by $|t|$ the number of its internal vertices. We denote by $Y_{n}$ the set of planar binary trees with $n$ internal vertices, so that $Y=\bigcup_{n=0}^{\infty} Y_{n}$.

Let $\mathcal{H}^{\alpha, n c}$ be the non-commutative lift of the Hopf algebra $\mathcal{H}^{\alpha}$ introduced in $[1,2]$ to describe the renormalization of the electric charge in the perturbative expansion of quantum electrodynamics based on planar binary trees. It is the graded and connected Hopf algebra $\mathbb{Q} Y$ spanned by all planar binary trees, endowed with the product over, cf. [11]: given two planar binary trees $s, t \neq \mid$, the tree $s$ over $t$, denoted by $s / t$, is the tree obtained by grafting the root of $s$ over the left-most leaf of $t$, that is,

$$
s / t=\stackrel{s}{t} t
$$

This product is not commutative, and the root tree | is the unit. Moreover, any tree can be decomposed as the over product of subtrees which have nothing branched on

their left-most leaf. If we set $v(t)=Y^{t}$ to denote these trees, the algebra $\mathcal{H}^{\alpha, \mathrm{nc}}$ is in fact isomorphic to the free algebra $T(V)$, where $V=\operatorname{Span}\{v(t), t \in Y\}$.

The coproduct $\Delta^{\alpha}: \mathcal{H}^{\alpha, \mathrm{nc}} \rightarrow \mathcal{H}^{\alpha, \mathrm{nc}} \otimes \mathcal{H}^{\alpha, \mathrm{nc}}$ can be described in an elegant way in the form proposed by Palacios in her Master Thesis [14]. For this, we need some notation. Let us denote by $\vee: Y_{n} \times Y_{m} \rightarrow Y_{n+m+1}$ the operation which grafts two trees on a new root, that is

$$
s \vee t={ }^{s}{ }^{t}
$$

and call dressed comb the tree $\gamma\left(t_{1}, \ldots, t_{k}\right)$ recursively given by

$$
\begin{gathered}
\gamma(t)=t \vee 1={ }^{t} Y, \\
\gamma\left(t_{1}, t_{2}, \ldots, t_{k}\right)=t_{1} \vee \gamma\left(t_{2}, \ldots, t_{k}\right)={ }^{t_{1}} t^{t_{2}} \cdot \dot{t_{k}}
\end{gathered}
$$


Then, any tree $t \neq \mid$ can be written as $t=\gamma\left(t_{1}, t_{2}, \ldots, t_{k}\right)$ for some suitable trees $t_{i}$.

The coproduct $\Delta^{\alpha}$ is the unital algebra homomorphism defined on the generators as

$$
\begin{aligned}
\Delta^{\alpha} Y & =|\otimes Y+Y \otimes|, \\
\Delta^{\alpha} v(t) & =\mid \otimes v(t)+\sum v\left(\gamma\left(t_{1(1)}, t_{2(1)}, \ldots, t_{k(1)}\right)\right) \otimes t_{1(2)} / t_{2(2)} / \cdots / t_{k(2)},
\end{aligned}
$$

where $t=\gamma\left(t_{1}, t_{2}, \ldots, t_{k}\right)$ and $\sum t_{i(1)} \otimes t_{i(2)}=\Delta^{\alpha}\left(t_{i}\right)$ is the Sweedler notation for the coproduct applied to each subtree $t_{i}$.

Finally, the counit $\varepsilon: \mathcal{H}^{\alpha, \mathrm{nc}} \rightarrow \mathbb{Q}$ is the unital algebra morphism with value $\varepsilon(v(t))=0$ on the generators.

Let $\left(\mathcal{H}^{\alpha, \mathrm{nc}}\right)^{*}=T\left(V^{*}\right)$ be its dual Hopf algebra, with primitive elements given by $V^{*}=\operatorname{Span}\left\{v\left(t^{*}\right), t \in Y\right\}$, where $t^{*}$ is the dual form of the tree $t$. Then $\left(\mathcal{H}^{\alpha, \mathrm{nc}}\right)^{*}$ is a right-sided cofree-coassociative $\mathrm{CHA}$, and $V^{*}$ is a brace algebra. We describe here its brace structure.

Theorem 4.1 For any $q>0$, the brace product $M_{1 q}$ on $V^{*}$ which induces the CHA structure on $\left(\mathcal{H}^{\text {dif,nc }}\right)^{*}$ is given by

$$
M_{1 q}\left(Y^{*} ; v\left(s_{1}\right)^{*} \cdots v\left(s_{q}\right)^{*}\right)=0
$$

and for any $t \neq \mid$ by

$$
M_{1 q}\left(v(t)^{*} ; v\left(s_{1}\right)^{*} \cdots v\left(s_{q}\right)^{*}\right)=\sum v\left(\gamma\left(p_{(1)}^{*}, \ldots, p_{(k)}^{*}\right)\right)^{*},
$$

where for $t=\gamma\left(t_{1}, \ldots, t_{k}\right)$ we set $p^{*}=\left(t_{1}^{*} \cdots t_{l}^{*}\right) \star\left(v\left(s_{1}\right)^{*} \cdots v\left(s_{q}\right)^{*}\right)$, and where we use the Sweedler notation

$$
\sum p_{(1)}^{*} \otimes p_{(2)}^{*} \otimes \cdots \otimes p_{(k)}^{*}=\left(\Delta^{*}\right)^{k-1} p^{*}
$$

for the deconcatenation coproduct $\Delta^{*}$ applied $k-1$ times to $p^{*}$.

Proof According to (2.13), if we denote $a^{*}=v\left(s_{1}\right)^{*} \cdots v\left(s_{q}\right)^{*}$, the brace product on $V^{*}$ can be computed as

$$
\begin{aligned}
M_{1 q}\left(v(t)^{*} ; a^{*}\right) & =\sum_{u \in Y}\left\langle v(t)^{*} \star a^{*} \mid v(u)\right\rangle v(u)^{*}=\sum_{u \in Y}\left\langle v(t)^{*} \otimes a^{*} \mid \Delta^{\alpha} v(u)\right\rangle v(u)^{*} \\
& =\left\langle v(t)^{*} \otimes a^{*}\left|\Delta^{\alpha} v(\mid)\right| v(\mid)^{*}+\sum_{u \neq \mid}\left\langle v(t)^{*} \otimes a^{*}\left|\Delta^{\alpha} v(u)\right| v(u)^{*} .\right.\right.
\end{aligned}
$$

The first term gives

$$
\left\langle v(t)^{*} \otimes a^{*}|Y \otimes|+|\otimes Y| Y^{*}=0\right.
$$

because $\left\langle a^{*}||\right\rangle=0$ for $q>0$, and $\left\langle v(t)^{*}||\right\rangle=0$ for any $t$. 
Then we suppose that $u \neq \mid$ and we evaluate the second term of (4.2) using the expression (4.1) for $\Delta^{\alpha} v(u)$. The first part gives

$$
\left\langle v(t)^{*} \otimes a^{*}|| \otimes v(u)\right\rangle=0
$$

for any choice of $t$.

The second part gives

$$
\begin{aligned}
& \left\langle v(t)^{*} \otimes a^{*} \mid v\left(\gamma\left(u_{1(1)}, u_{2(1)}, \ldots, u_{l(1)}\right)\right) \otimes u_{1_{(2)}} / u_{2(2)} / \cdots / u_{l(2)}\right\rangle \\
& \quad=\left\langle v(t)^{*} \mid v\left(\gamma\left(u_{1(1)}, u_{2(1)}, \ldots, u_{l(1)}\right)\right)\right\rangle\left\langle a^{*} \mid u_{1_{(2)}} / u_{2(2)} / \cdots / u_{l(2)}\right\rangle
\end{aligned}
$$

where we write $u$ as $\gamma\left(u_{1}, \ldots, u_{l}\right)$. The only primitive element $v(t)$ which can not be of the form $v\left(\gamma\left(u_{1(1)}, u_{2(1)}, \ldots, u_{k(1)}\right)\right)$ is $v(\mid)=Y$. In this case, the brace product is obviously

$$
M_{1 q}\left(Y^{*} ; a^{*}\right)=0
$$

for any $q>0$. Finally, let us consider the case $t \neq \mid$. Then $t=\gamma\left(t_{1}, \ldots, t_{k}\right)$, and the first pairing of (4.3) is non-zero only if

$$
\gamma\left(u_{1(1)}, u_{2(1)}, \ldots, u_{l(1)}\right)=t=\gamma\left(t_{1}, \ldots, t_{k}\right),
$$

that is, if and only if $l=k$ and

$$
u_{1(1)}=t_{1}, \quad u_{2(1)}=t_{2}, \quad \ldots, \quad u_{k(1)}=t_{k} .
$$

Therefore we have

$$
\begin{aligned}
& M_{1 q}\left(v(t)^{*} ; a^{*}\right) \\
& \left.=\sum_{u_{1}, \ldots, u_{k}}\left\langle v(t)^{*} \mid v\left(\gamma\left(u_{1(1)}, \ldots, u_{k(1)}\right)\right)\right\rangle\left\langle a^{*}\right| u_{1(2)} / \cdots / u_{l(2)}\right) v\left(\gamma\left(u_{1}, \ldots, u_{k}\right)\right)^{*} \\
& \left.=\sum_{u_{1}, \ldots, u_{k}}\left\langle t_{1}^{*} \cdots t_{k}^{*} \mid u_{1(1)} \cdots u_{k(1)}\right\rangle\left\langle a^{*}\right| u_{1(2)} / \cdots / u_{l(2)}\right) v\left(\gamma\left(u_{1}, \ldots, u_{k}\right)\right)^{*} \\
& =\sum_{u_{1}, \ldots, u_{k}}\left\langle t_{1}^{*} \cdots t_{k}^{*} \otimes a^{*} \mid \Delta^{\alpha}\left(u_{1} / \cdots / u_{k}\right)\right\rangle v\left(\gamma\left(u_{1}, \ldots, u_{k}\right)\right)^{*} \\
& =\sum_{u_{1}, \ldots, u_{k}}\left\langle\left(t_{1}^{*} \cdots t_{k}^{*}\right) \star a^{*} \mid u_{1} / \cdots / u_{k}\right\rangle v\left(\gamma\left(u_{1}, \ldots, u_{k}\right)\right)^{*} .
\end{aligned}
$$

Now, note that the deconcatenation coproduct $\Delta^{*}$ in $\left(\mathcal{H}^{\alpha, n c}\right)^{*}$ is dual to the product / in $\mathcal{H}^{\alpha, \mathrm{nc}}$, that is

$$
\left\langle a^{*} \mid b / c\right\rangle=\left\langle\Delta^{*}\left(a^{*}\right) \mid b \otimes c\right\rangle .
$$

Let us call $p^{*}=\left(t_{1}^{*} \cdots t_{k}^{*}\right) \star a^{*}$. If we apply $k-1$ times the deconcatenation coproduct $\Delta^{*}$ to $p^{*}$, and we use the Sweedler notation $\sum p_{(1)}^{*} \otimes p^{*}{ }_{(2)} \otimes \cdots \otimes p^{*}{ }_{(l)}=$ 
$\left(\Delta^{*}\right)^{k-1} p^{*}$, we obtain the final result

$$
\begin{aligned}
& M_{1 q}\left(v(t)^{*} ; a^{*}\right) \\
& \left.\quad=\sum_{u_{1}, \ldots, u_{k}} \sum\left\langle p_{(1)}^{*} \otimes p_{(2)}^{*} \otimes \cdots \otimes p^{*}{ }_{(k)}\right| u_{1} \otimes \cdots \otimes u_{k}\right) v\left(\gamma\left(u_{1}, \ldots, u_{k}\right)\right)^{*} \\
& \quad=\sum v\left(\gamma\left(p_{(1)}^{*}, \ldots, p_{(k)}^{*}\right)\right)^{*} .
\end{aligned}
$$

Examples 4.2 The simplest example is given by $M_{11}\left(Y^{*} ; Y^{*}\right)$. We have $Y=$ $v(Y)=v(\gamma(\mid))$, so that $k=1$ and $t_{1}=\mid$. Therefore, $p^{*}=1^{*} \star Y^{*}=Y^{*}$ and

$$
M_{11}\left(Y^{*} ; Y^{*}\right)=v\left(\gamma\left(Y^{*}\right)\right)=v\left(Y^{*}\right)=Y^{*}
$$

Similarly

$$
M_{11}\left(Y^{*} ; Y^{*}\right)=v\left(\gamma\left(Y^{*}\right)\right)=v\left(Y^{*}\right)=Y^{*}
$$

Another simple example is given by $M_{11}\left(Y^{*} ; Y^{*}\right)$. We have $Y=v(\gamma(Y))$, so that $k=1$ and $t_{1}=Y$. Since for any $t$ formula (2.13) gives

$$
Y^{*} \star v(t)^{*}=v(t)^{*} Y^{*}+Y^{*} v(t)^{*}+M_{1 q}\left(Y^{*} ; v(t)^{*}\right)=v(t)^{*} Y^{*}+Y^{*} v(t)^{*},
$$

because $M_{1 q}\left(Y^{*} ; v(t)^{*}\right)=0$, we get $p^{*}=Y^{*} \star Y^{*}=2 Y^{*}$, and finally

$$
M_{11}\left(Y^{*} ; Y^{*}\right)=2 v(\gamma(Y))^{*}=2 v(Y)^{*}=2 Y^{*}
$$

Now let us compute $M_{11}\left(Y^{*} ; Y^{*}\right)$. We have $Y^{Y}=v(\gamma(||)$,$) so that k=2$ and $t_{1}=t_{2}=\mid$. Since $\left.\left.\right|^{*}\right|^{*}=1^{*}$, we have $p^{*}=1^{*} \star Y^{*}=Y^{*}$ and therefore

$$
\begin{aligned}
M_{11}\left(Y^{*} ; Y^{*}\right) & =v(\gamma(Y, \mid))^{*}+v(\gamma(\mid, Y))^{*}=v(Y)^{*}+v(Y)^{*} \\
& =Y^{*}+Y^{*} .
\end{aligned}
$$

Finally, let us compute an example of $M_{1 q}$ with $q>1$. We consider $M_{12}\left(Y^{*} ; Y^{*} Y^{*}\right)$. We have $Y=v(\gamma(\mid))$, so that $k=1$ and $t_{1}=\mid$. Therefore, $p^{*}=1^{*} \star\left(Y^{*} Y^{*}\right)=Y^{*}$ and

$$
M_{12}\left(Y^{*} ; Y^{*} Y^{*}\right)=v(\gamma(Y))^{*}=v(Y)^{*}=Y^{*} .
$$

Similarly

$$
\begin{aligned}
M_{12}\left(Y^{*} ; Y^{*} Y^{*}\right) & =v(\gamma(Y, \mid))^{*}+v(\gamma(Y, Y))^{*}+v(\gamma(\mid, Y))^{*} \\
& =v(Y Y)^{*}+v(Y Y)^{*}+v(Y)^{*}=Y^{*}+Y^{*}+Y^{*} .
\end{aligned}
$$


Remark 4.3 The brace structure can be described as follows:

$$
M_{1 q}\left(v(t)^{*} ; v\left(s_{1}\right)^{*} \cdots v\left(s_{q}\right)^{*}\right)=\sum_{u} v(u)^{*},
$$

where the sum runs over any tree $u$ obtained by branching the trees $v\left(s_{i}\right)$ or any block $v\left(s_{i}\right) / \cdots / v\left(s_{j}\right)$ on the $\backslash$-leaves of $t$ or inside the $\backslash$-branches of $t$, by preserving the order from left to right of the trees $v\left(s_{i}\right)$ and of the $\backslash$-branches of $t$.

\section{The right-sided combinatorial structure of the Hopf algebra $T(\bar{T}(B))^{*}$}

In the paper [4], Brouder and Schmitt introduced a non-commutative version $\mathcal{H}$ of a renormalization Hopf algebra inspired by Pinter's work on the Epstein-Glaser renormalization of quantum fields on the configuration space. In this section we describe the brace structure on the set of primitive elements of its dual Hopf algebra $\mathcal{H}^{*}$.

As an algebra, $\mathcal{H}$ is the tensor algebra $T(V)$ on a set of generators $V=\bar{T}(B)=$ $\bigoplus_{n \geq 1} B^{\otimes n}$ which is the augmentation ideal of the tensor algebra over a given bialgebra $B$.

In order to apply the dualization procedure described in Sect. 2, we have to assume that the bialgebra $B$ admits at most a countable basis, and that this has been fixed. Let us denote by $\left\{x_{n}\right\}$ the chosen basis of $B$. We also denote the product (in $B$ ) by a dot, namely $x \cdot y$ for any $x, y \in B$, and the coproduct (in $B$ ) through the Sweedler notation $\delta(x)=\sum x_{(1)} \otimes x_{(2)}$.

In $V=\bar{T}(B)$, we denote the tensor products by tuples. Therefore the elements $v_{n_{1}, \ldots, n_{k}}=\left(x_{n_{1}}, \ldots, x_{n_{k}}\right)$ form a basis of $V$. On $V$ we consider two coproducts. The first one is the extension of the coproduct on $B$ as an algebra morphism, namely

$$
\delta(x, y, \ldots, z)=\sum\left(x_{(1)}, y_{(1)}, \ldots, z_{(1)}\right) \otimes\left(x_{(2)}, y_{(2)}, \ldots, z_{(2)}\right) .
$$

For a generic element $v$ of $V$, we use the same Sweedler notation $\delta(v)=\sum v_{(1)} \otimes v_{(2)}$ as on $B$. The second coproduct is the (reduced) deconcatenation coproduct

$$
\Delta(x, y, \ldots, z)=(x) \otimes(y, \ldots, z)+(x, y) \otimes(\ldots, z)+\cdots+(x, y, \ldots) \otimes(z) .
$$

For this coproduct we use a modified Sweedler notation $\Delta(v)=\sum v_{[1]} \otimes v_{[2]}$.

Finally, in $\mathcal{H}=T(V)$, we denote the tensor product by the concatenation. A generic element in $\mathcal{H}$ is then of the form $a=u v \cdots w$, where $u, v, \ldots, w \in V$, and for any elements $a, b$ we denote their product (in $T(V)$ ) by $a b$. Both coproducts $\delta$ and $\Delta$ can be extended to $\mathcal{H}$. The renormalization coproduct is then the algebra homomorphism defined on a generator $v \in V$ as

$$
\Delta^{\mathcal{H}}(v)=\sum_{n=1}^{\infty} \sum\left(\mu\left(v_{[1](1)}\right), \ldots, \mu\left(v_{[n](1)}\right)\right) \otimes v_{[1](2)} \cdots v_{[n](2)},
$$

where $\sum v_{[1]} \otimes \cdots \otimes v_{[n]}=\Delta^{n-1}(v)$ is the result of the deconcatenation applied $n-$ 1 times to $v$, and $\mu: \bar{T}(B) \rightarrow B, \mu(x, y, \ldots, z)=x \cdot y \cdots z$ is the product of $B$ extended to many factors. 
Note that for any $v \in V$, the left tensor factor $\left(\mu\left(v_{[1](2)}\right), \ldots, \mu\left(v_{[n](2)}\right)\right)$ of $(5.1)$ belongs to $V$ (in fact it belongs to $B^{\otimes n}$ ), while the right factor $v_{[1](1)} \cdots v_{[n](1)}$ belongs to $V^{\otimes n}$, therefore $\Delta^{\mathcal{H}}$ cannot be defined in $V$.

Note also that any monomial of elements of $B$ in $v \in V$ has finite length. Since the deconcatenation of $v$ vanishes if repeated more then the length of $v$, the sum over $n$ in formula (5.1) is indeed finite. For example, let us compute the simplest coproducts, for $x, y$ and $z$ in $B$ :

$$
\begin{aligned}
\Delta^{\mathcal{H}}(x)= & \sum\left(x_{(1)}\right) \otimes\left(x_{(2)}\right) \\
\Delta^{\mathcal{H}}(x, y)= & \sum\left(x_{(1)}, y_{(1)}\right) \otimes\left(x_{(2)}\right)\left(y_{(2)}\right)+\sum\left(x_{(1)} \cdot y_{(1)}\right) \otimes\left(x_{(2)}, y_{(2)}\right) \\
\Delta^{\mathcal{H}}(x, y, z)= & \sum\left(x_{(1)}, y_{(1)}, z_{(1)}\right) \otimes\left(x_{(2)}\right)\left(y_{(2)}\right)\left(z_{(2)}\right) \\
& +\sum\left(x_{(1)}, y_{(1)} \cdot z_{(1)}\right) \otimes\left(x_{(2)}\right)\left(y_{(2)}, z_{(2)}\right) \\
& +\sum\left(x_{(1)} \cdot y_{(1)}, z_{(1)}\right) \otimes\left(x_{(2)}, y_{(2)}\right)\left(z_{(2)}\right) \\
& +\sum\left(x_{(1)} \cdot y_{(1)} \cdot z_{(1)}\right) \otimes\left(x_{(2)}, y_{(2)}, z_{(2)}\right) .
\end{aligned}
$$

It is important to remark that, if $v=\left(x_{n_{1}}, \ldots, x_{n_{l}}\right)$, then $\Delta^{\mathcal{H}} v$ is a sum of elements of $B^{\otimes k}$ tensorized by elements of $\bar{T}(B)^{\otimes k}$, for $k=1$ to $l$.

We now consider the dual Hopf algebra $\mathcal{H}^{*}=T\left(V^{*}\right)$, where $V^{*}$ is the graded dual space $\bar{T}\left(B^{*}\right)$. Here $B^{*}$ is the dual bialgebra of $B$, with basis elements $x_{m}^{*}$ such that $\left\langle x_{m}^{*} \mid x_{n}\right\rangle=\delta_{m, n}$. The product and coproduct in $B^{*}$ are defined in terms of those of $B$ by the standard duality

$$
\begin{aligned}
\left\langle x^{*} \cdot y^{*}, z\right\rangle & =\left\langle x^{*} \otimes y^{*}, \delta z\right\rangle, \\
\left\langle\delta x^{*}, y \otimes z\right\rangle & =\left\langle x^{*}, y \cdot z\right\rangle .
\end{aligned}
$$

In $V^{*}=\bar{T}\left(B^{*}\right)$ we have $\left(x_{1}, \ldots, x_{n}\right)^{*}=\left(x_{1}^{*}, \ldots, x_{n}^{*}\right)$ and therefore

$$
\left\langle\left(x_{1}^{*}, \ldots, x_{n}^{*}\right) \mid\left(y_{1}, \ldots, y_{m}\right)\right\rangle=\delta_{n, m}\left\langle x_{1}^{*} \mid y_{1}\right\rangle \cdots\left\langle x_{n}^{*} \mid y_{n}\right\rangle .
$$

As we said, $\mathcal{H}^{*}$ is a right-sided cofree-coassociative $\mathrm{CHA}$, with deconcatenation coproduct $\Delta^{*}$ and product $\star$. In this section we describe the brace product induced by $\star$ on the set $V^{*}$ of primitive elements of $\mathcal{H}^{*}$.

Theorem 5.1 The brace product $M_{1 q}$ on $V^{*}=\bar{T}\left(B^{*}\right)$ which induces the CHA structure on $\mathcal{H}^{*}$ is given on the elements $v^{*}=\left(x^{1}, \ldots, x^{n}\right)^{*}$ and $u_{1}^{*}, \ldots, u_{q}^{*}$, for any $q>0$, by

$$
M_{1 q}\left(v^{*} ; u_{1}^{*} \cdots u_{q}^{*}\right)=0, \quad \text { if } n \neq q,
$$

and, for $n=q$, by

$$
M_{1 q}\left(v^{*} ; u_{1}^{*} \cdots u_{q}^{*}\right)=\left(x_{(1)}^{1} \cdot y_{1}^{1}, \ldots, x_{\left(k_{1}\right)}^{1} \cdot y_{1}^{k_{1}}, \ldots, x_{(1)}^{q} \cdot y_{q}^{1}, \ldots, x_{\left(k_{q}\right)} \cdot y_{q}^{k_{q}}\right)^{*},
$$

where $u_{i}^{*}=\left(y_{i}^{1}, \ldots, y_{i}^{k_{i}}\right)^{*}$, for $i=1, \ldots, q$. 
Proof If $n \neq q$, we already remarked in the expression (5.1) of the coproduct that no term can occur.

If $n=q$, consider $v=\left(x^{1}, \ldots, x^{q}\right) \in B^{\otimes q}$ and $u_{1}, \ldots, u_{q}$ in $B^{\otimes k_{1}}, \ldots, B^{\otimes k_{q}}$, respectively. Set $a=u_{1} \cdots u_{q} \in V^{\otimes q}$. Then

$$
\begin{aligned}
M_{1 q}\left(v^{*} ; a^{*}\right) & =\sum_{k} \sum_{w \in B^{\otimes k}}\left\langle v^{*} \otimes a^{*}\left|\Delta^{\mathcal{H}} w\right| w^{*}\right. \\
& =\sum_{k} \sum_{w \in B^{\otimes k}} \sum\left\langle v^{*} \otimes a^{*} \mid w_{(1)} \otimes w_{(2)}\right\rangle w^{*},
\end{aligned}
$$

where the sums run over the generators $w$ of $B^{\otimes k}$, that is, elements of the form $w=\left(z^{1}, \ldots, z^{k}\right)$ where $z^{i}$ are generators of $B$, and where $\sum w_{(1)} \otimes w_{(2)}=\delta(w)$ denotes the coproduct of $B$.

As a consequence, the term corresponding to $w$ appears in $M_{1 q}\left(v^{*} ; a^{*}\right)$ if $w_{(1)}=v$ and $w_{(2)}=a$. The equality $w_{(1)}=v=\left(x^{1}, \ldots, x^{q}\right)$ can be rewritten

$$
\begin{aligned}
z_{(1)}^{1} \cdots z_{(1)}^{k_{1}} & =x^{1}, \\
z_{(1)}^{k_{1}+1} \cdots z_{(1)}^{k_{1}+k_{2}} & =x^{2}, \\
& \cdots \\
z_{(1)}^{k_{1}+\cdots+k_{q-1}+1} \cdots z_{(1)}^{k} & =x^{q} .
\end{aligned}
$$

The equality $w_{(2)}=a=u_{1} \cdots u_{q}$ can be rewritten

$$
\begin{aligned}
\left(z_{(2)}^{1}, \ldots, z_{(2)}^{k_{1}}\right) & =u_{1}, \\
\left(z_{(2)}^{k_{1}+1}, \ldots, z_{(2)}^{k_{1}+k_{2}}\right) & =u_{2}, \\
& \ldots \\
\left(z_{(2)}^{k_{1}+\cdots+k_{q-1}+1}, \ldots, z_{(2)}^{k}\right) & =u_{q} .
\end{aligned}
$$

The second equality means that $k=k_{1}+\cdots+k_{q}$. Because of this special form, each block can be treated independently.

We consider the first block and we write $k=k_{1}$ and $u_{1}=\left(y_{1}, \ldots, y_{k}\right)$. We have now, for the first block and after putting $x=x^{1}$,

$$
\begin{aligned}
X & =\sum_{z^{1}, \ldots, z^{k}}\left\langle\left(x^{*}\right) \otimes\left(y_{1}, \ldots, y_{k}\right)^{*},\left(z_{(1)}^{1} \cdots z_{(1)}^{k}\right) \otimes\left(z_{(2)}^{1}, \ldots, z_{(2)}^{k}\right)\right\rangle\left(z^{1}, \ldots, z^{k}\right)^{*} \\
& =\sum_{z^{1}, \ldots, z^{k}}\left\langle\left(x^{*}\right),\left(z_{(1)}^{1} \cdots z_{(1)}^{k}\right)\right\rangle\left\langle\left(y_{1}, \ldots, y_{k}\right)^{*},\left(z_{(2)}^{1}, \ldots, z_{(2)}^{k}\right)\right\rangle\left(z^{1}, \ldots, z^{k}\right)^{*} \\
& =\sum_{z^{1}, \ldots, z^{k}}\left\langle x_{(1)}^{*}, z_{(1)}^{1}\right\rangle \cdots\left\langle x_{(k)}^{*}, z_{(1)}^{k}\right)\left\langle y_{1}^{*}, z_{(2)}^{1}\right\rangle \cdots\left\langle y_{k}^{*}, z_{(2)}^{k}\right\rangle\left(z^{1}, \ldots, z^{k}\right)^{*},
\end{aligned}
$$


where we used the fact that the coproduct in $B^{*}$ is the dual of the product in $B$. Now we use the fact that the product in $B^{*}$ is the dual of the coproduct in $B$, and obtain

$$
\begin{aligned}
X & =\sum_{z^{1}, \ldots, z^{k}}\left\langle x_{(1)}^{*} \cdot y_{1}^{*}, z^{1}\right\rangle \cdots\left\langle x_{(k)}^{*} \cdot y_{k}^{*}, z^{k}\right\rangle\left(z^{1}, \ldots, z^{k}\right)^{*} \\
& =\sum\left(x_{(1)} \cdot y_{1}, \ldots, x_{(k)} \cdot y_{k}\right)^{*} .
\end{aligned}
$$

The same is done for each block and we finally find

$$
M_{1 q}\left(v^{*} ; a^{*}\right)=\left(x_{(1)}^{1} \cdot y_{1}^{1}, \ldots, x_{\left(k_{1}\right)}^{1} \cdot y_{k_{1}}^{1}, \ldots, x_{(1)}^{q} \cdot y_{1}^{q}, \ldots, x_{(k)}^{q} \cdot y_{k_{q}}^{q}\right)^{*} .
$$

Examples 5.2 Examples of $M_{11}$ are:

$$
\begin{aligned}
M_{11}\left(\left(x^{*}\right) ;\left(y^{*}\right)\right) & =\left(x^{*} \cdot y^{*}\right), \\
M_{11}\left(\left(x^{*}\right) ;\left(y^{*}, z^{*}\right)\right) & =\sum\left(x^{*}{ }_{(1)} \cdot y^{*}, x^{*}{ }_{(2)} \cdot z^{*}\right), \\
M_{11}\left(\left(x^{*}\right) ;\left(y_{1}^{*}, \ldots, y_{n}^{*}\right)\right) & =\left(x^{*}{ }_{(1)} \cdot y_{1}^{*}, \ldots, x_{(n)}^{*} \cdot y_{n}^{*}\right) .
\end{aligned}
$$

Examples of $M_{12}$ are:

$$
\begin{aligned}
& M_{12}\left((x, y)^{*} ;(s)^{*}(t)^{*}\right)=(x \cdot s, y \cdot t)^{*}, \\
& M_{12}\left((x, y)^{*} ;(s)^{*}\left(t_{1}, t_{2}\right)^{*}\right)=\sum\left(x \cdot s, y_{(1)} \cdot t_{1}, y_{(2)} \cdot t_{2}\right)^{*}, \\
& M_{12}\left((x, y)^{*} ;\left(s_{1}, s_{2}\right)^{*}(t)^{*}\right)=\sum\left(x_{(1)} \cdot s_{1}, x_{(2)} \cdot s_{2}, y \cdot t\right)^{*}, \\
& M_{12}\left(\left(x^{*}, y^{*}\right) ;\left(s_{1}^{*}, \ldots, s_{m}^{*}\right)\left(t_{1}^{*}, \ldots, t_{n}^{*}\right)\right)=\sum\left(x_{(1)}^{*} \cdot s_{1}^{*}, \ldots, x_{(m)}^{*} \cdot s_{m}^{*},\right. \\
&\left.y^{*}{ }_{(1)} \cdot t_{1}^{*}, \ldots, y_{(n)}^{*} \cdot t_{n}^{*}\right) .
\end{aligned}
$$

\section{References}

1. Brouder, Ch., Frabetti, A.: Renormalization of QED with planar binary trees. Eur. Phys. J. C 19, 715-741 (2001)

2. Brouder, Ch., Frabetti, A.: QED Hopf algebras on planar binary trees. J. Algorithms 267, 298-322 (2003)

3. Brouder, Ch., Frabetti, A., Krattenthaler, Ch.: Non-commutative Hopf algebra of formal diffeomorphisms. Adv. Math. 200, 479-524 (2006)

4. Brouder, Ch., Schmitt, W.: Renormalization as a functor on bialgebras. J. Pure Appl. Algebra 209, 477-495 (2007)

5. Chapoton, F.: Un théorème de Cartier-Milnor-Moore-Quillen pour les bigèbres dendriformes et les algèbres brace. J. Pure Appl. Algebra 168(1), 1-18 (2002)

6. Foissy, L.: Les algèbres de Hopf des arbres enracinés décorés, II. Bull. Sci. Math. 126, 249-288 (2002)

7. Grossman, R., Larson, R.G.: Hopf-algebraic structure of families of trees. J. Algebra 126, 184-210 (1989)

8. Guin, D., Oudom, J.-M.: On the Lie envelopping algebra of a pre-Lie algebra. K-Theory 2(1), 147167 (2008) 
9. Kreimer, D.: On the Hopf algebra structure of perturbative quantum field theories. Adv. Theor. Math. Phys. 2, 303-334 (1998)

10. Loday, J.-L., Ronco, M.: Hopf algebra of the planar binary trees. Adv. Math. 139, 293-309 (1998)

11. Loday, J.-L.: Arithmetree. J. Algorithms 258, 275-309 (2002)

12. Loday, J.-L., Ronco, M.: On the structure of cofree Hopf algebras. J. Reine Angew. Math. 592, 123155 (2006)

13. Loday, J.-L., Ronco, M.: Combinatorial Hopf algebras. In: Clay Mathematical Proceeding (to appear)

14. Palacios, P.: Una generalización a operads de la construcción de Hopf de Connes-Kreimer. Tesis de licenciatura, Universidad de Buenos Aires, Argentina (2002)

15. Ronco, M.: Eulerian idempotents and Milnor-Moore theorem for certain non cocommutative Hopf algebras. J. Algorithms 254, 152-172 (2002)

16. van der Laan, P.: Operads and the Hopf algebras of renormalization. Preprint (2003), http://www. arxiv.org/abs/math-ph/0311013 